\title{
Research Article \\ Relation between Press Intensity and Angular Velocity at a RPPP Mechanism
}

\author{
Şenay Baydaş ${ }^{1}$ and Bülent Karakaş ${ }^{2}$ \\ ${ }^{1}$ Department of Mathematics, Faculty of Science and Arts, Yüzüncü Yıl University, 65080 Van, Turkey \\ ${ }^{2}$ Department of Business Administration, Faculty of Economics and Administrative Science, \\ Yüzüncü Yil University, 65080 Van, Turkey \\ Correspondence should be addressed to Şenay Baydaş, senay.baydas@gmail.com
}

Received 9 June 2010; Accepted 3 February 2011

Academic Editor: Bin Liu

Copyright (c) 2011 Ş. Baydaş and B. Karakaş. This is an open access article distributed under the Creative Commons Attribution License, which permits unrestricted use, distribution, and reproduction in any medium, provided the original work is properly cited.

\begin{abstract}
We study some properties of RPPP. RPPP is discussed by rising with constant velocity along a given axis. The constant pressure which it stresses on a constant axis is defined by the increasing PPP. Some relations between the increase at PPP and angular velocity at R are analyzed and relations of correlation are investigated at Matlab.
\end{abstract}

\section{Introduction}

Mechanisms may have different types of joints, such as linear, rotary, sliding, or spherical. Although spherical joints are common in many systems, since they posses multiple degrees of freedom, and thus, are difficult to control, spherical joints are not common in robotics, except in research. Most robots have either a linear (prismatic) joint or a rotary (revolute) joint [1]. To describe the translational and rotational relationships between adjacent links, Denavit and Hartenberg proposed a matrix method of systematically establishing a coordinate system to each link of an articulated chain. The Denavit-Hartenberg (D-H) representation results in a $4 \times 4$ homogeneous transformation matrix representing each link's coordinate system at the joint with respect to the previous link's coordinate system. Thus, through sequential transformations, the end effector expressed in the hand coordinates can be transformed and expressed in the base coordinates which make up the inertial frame of this dynamic system [2].

Calculating the position and orientation of the hand of robot is called forward kinematic analysis. In other words, if all robot joint variables are known, using forward kinematic equations, one can calculate where the robot is at any instant [1]. 


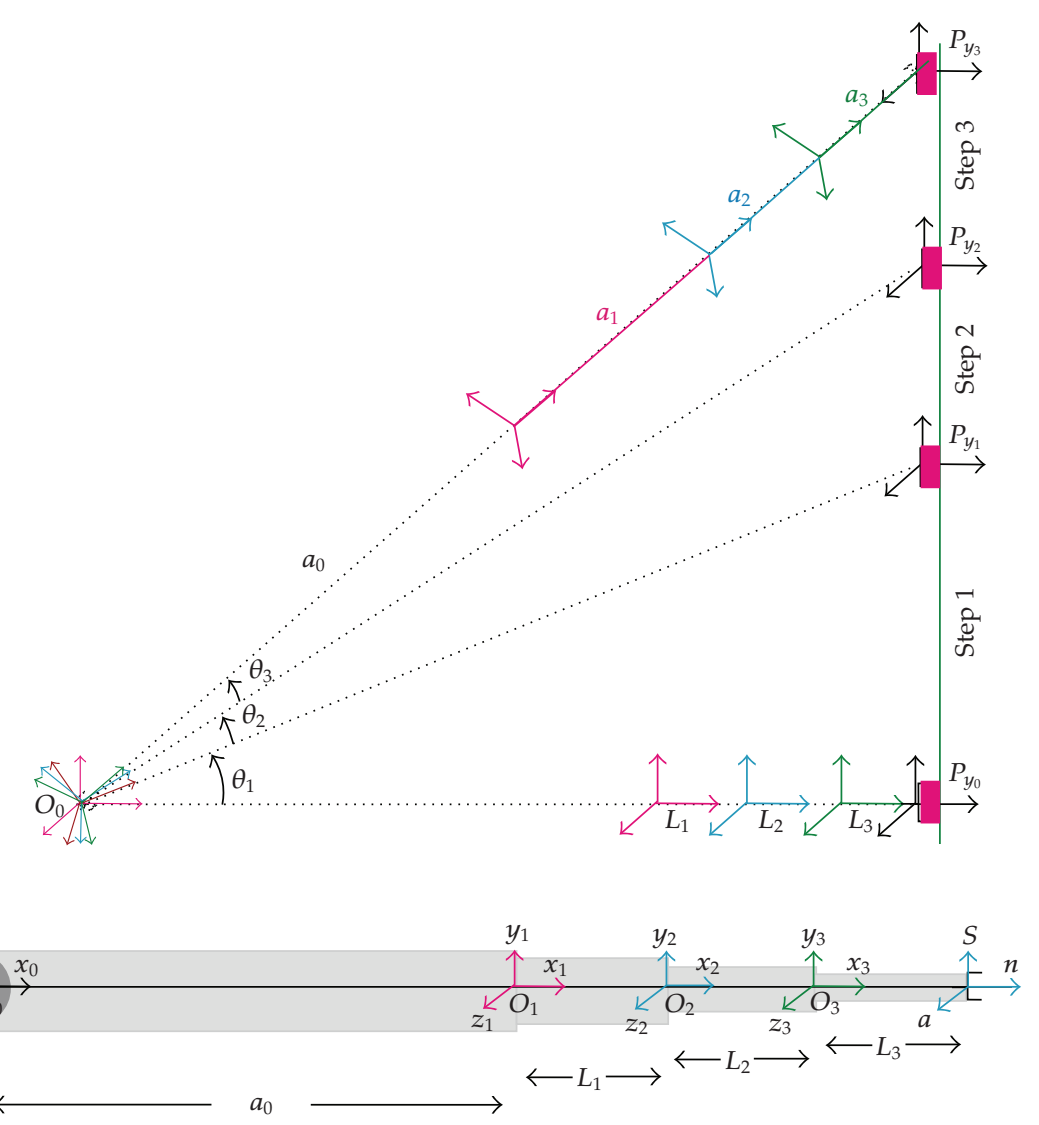

Figure 1: Settle of frames and end effector according to steps.

A robot manipulator is often designed as spatial open chain in which each joint is actuated. The kinematics equations of the open chain define the position of the end effector for a given set of values for the joint parameters. It is also necessary to be able to compute the joint parameter values that provide a desired position for end effector. This is known as the inverse kinematics problem in robotics [3].

Three serial chains with 3-dof each connect the fixed link to the end effector link $[4,5]$.

Mechanism of RPPP robot is studied in this paper. This mechanism is used for rising with constant velocity along fixed axis at screw plane. Design and equations of matrix of mechanisms are given in Section 2.

\section{Presentation of RPPP}

RPPP mechanism and its special tasks are dealt with in this paper. The first main function is to rise with a constant velocity of the end effector at a constant direction. Its other functions are to come back and to displace the constant axis (Figures 1 and 2).

At the rising and returning time, a constant pressure is done to the constant axis. The pressure is fixed if pistons have an expressed pressure at any time. Firstly let us write kinematic equations for RPPP. 


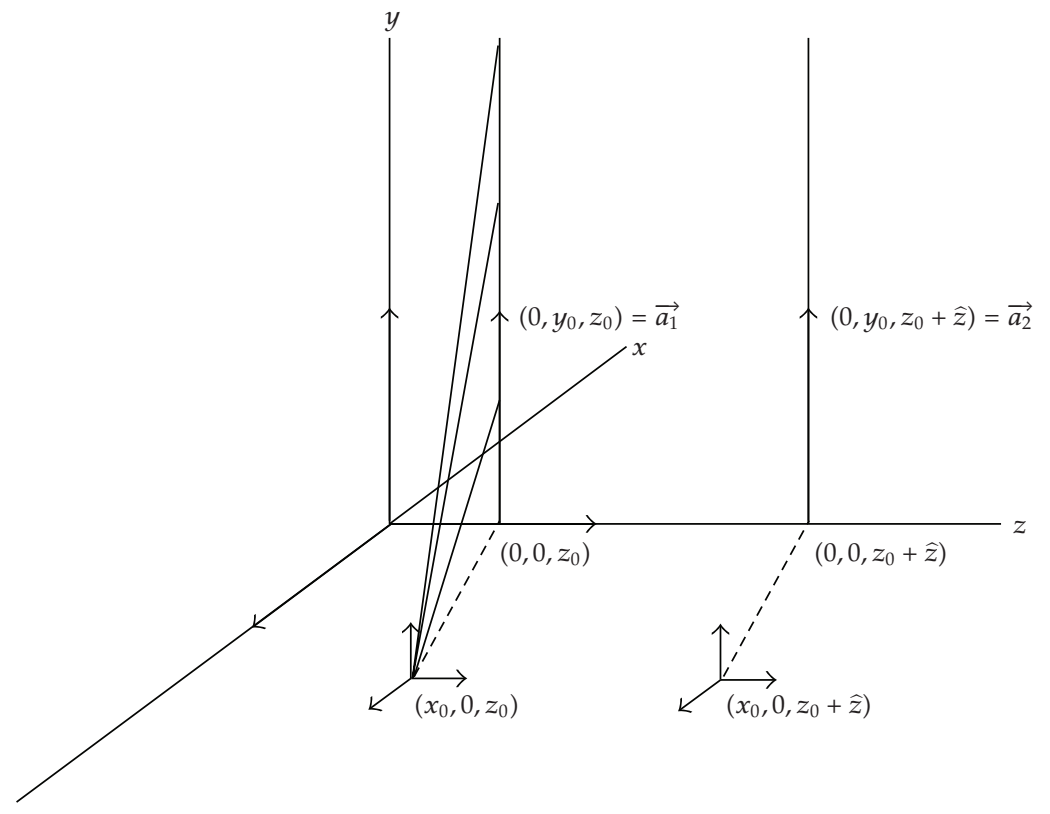

Figure 2: Skid motion.

The frames of mechanism which we investigated can be seen in Figure 1, where

$$
L=a_{0}+L_{1}+L_{2}+L_{3}
$$

$a_{1}, a_{2}$, and $a_{3}$ are latest lengths of $L_{1}, L_{2}, L_{3} . a_{1}, a_{2}$, and $a_{3}$ are known as

$$
\begin{gathered}
a_{1}=L\left(\sqrt{1+\tan ^{2} \theta_{1}}-1\right), \\
a_{2}=L\left(\sqrt{1+\tan ^{2}\left(\theta_{1}+\theta_{2}\right)}-1\right)-a_{1}, \\
a_{3}=L\left(\sqrt{1+\tan ^{2}\left(\theta_{1}+\theta_{2}+\theta_{3}\right)}-1\right)-\left(a_{1}+a_{2}\right) .
\end{gathered}
$$

$a_{i}$ is a function of $\theta_{i}$ and $\theta_{i}$ is a function of $a_{i}$ too, in Figure 3. Point values at the constant axis for $\theta_{i}$ are as follows.

$$
\begin{gathered}
M_{i}=\left\|O^{\prime} P y_{i}\right\|=L \tan \left(\sum_{j=1}^{i} \theta_{j}\right), \quad 1 \leq i \leq 3, \\
M_{1}=\left\|O^{\prime} P y_{0}\right\|=L \tan \left(\theta_{1}\right), \\
M_{2}=L \tan \left(\theta_{1}+\theta_{2}\right), \\
M_{3}=L \tan \left(\theta_{1}+\theta_{2}+\theta_{3}\right) .
\end{gathered}
$$



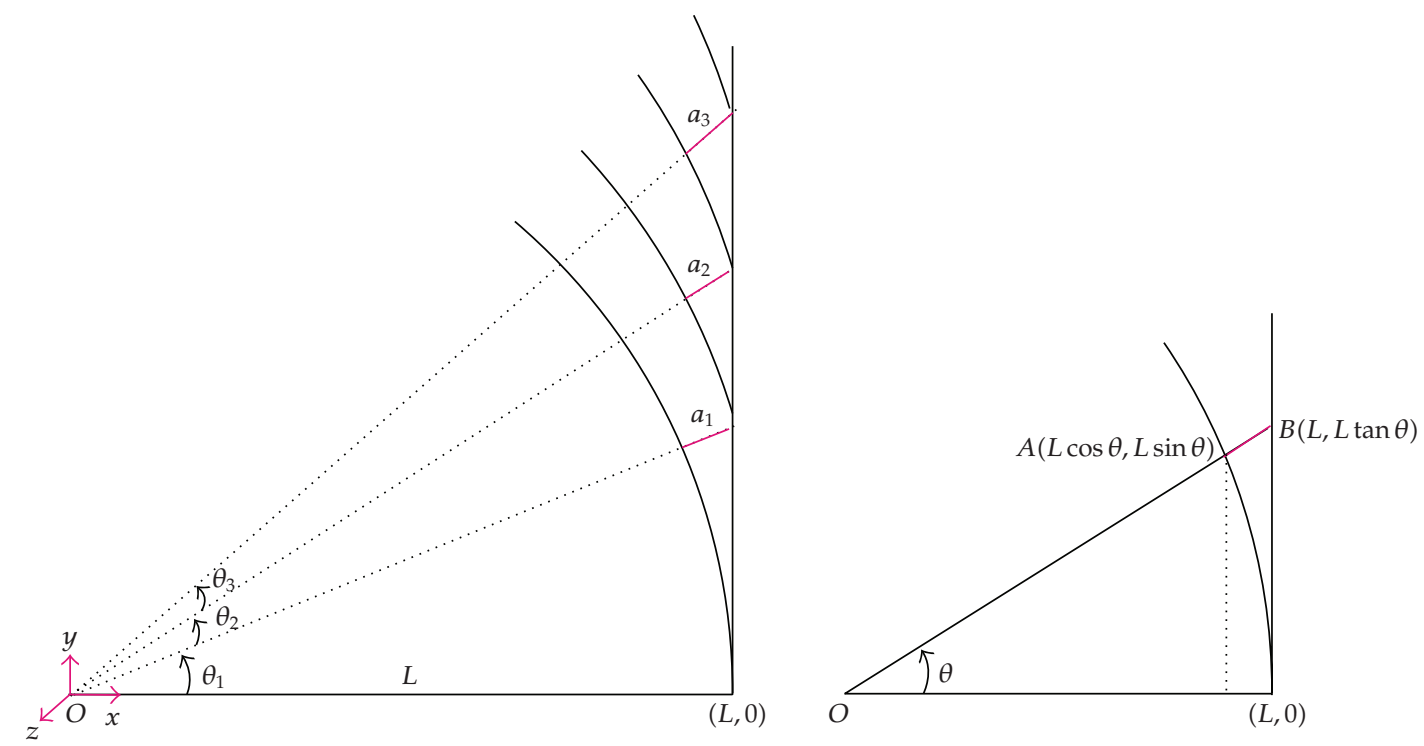

Figure 3: Relation between $\theta_{i}$ and $a_{i}$.

Table 1: D-H parameters.

\begin{tabular}{cccc}
\hline$\theta_{i}$ & $d_{i}$ & $a_{i}$ & $\alpha_{i}$ \\
$\theta_{1}$ & 0 & $a_{1}$ & 0 \\
$\theta_{2}$ & 0 & $a_{2}$ & 0 \\
$\theta_{3}$ & 0 & $a_{3}$ & 0 \\
\hline
\end{tabular}

The end effector rises with fixed speed along vector $\vec{a}=\left(0, y, z_{0}\right)$ when the base frame is at point $(0,0,0)$.

The turning back motion is an inverse of rising. The skid motion is a displacement of fixed frame from $\left(x_{0}, 0, z_{0}\right)$ to $\left(x_{0}, 0, z_{o}+\widehat{z}\right)$.

Now we can define the general procedure, based on Denavit-Hartenberg representation, to assign frames to each joint.

D-H parameters belonging to mechanism RPPP are calculated as in Table 1.

We wrote the motion matrix with a different method, instead of using the D-H parameters.

A translation vector according to Figure 4 is

$$
\overrightarrow{A B}=B-A=(L-L 2 \cos \theta, L \cos \theta-L \tan \theta) .
$$

Consequently we obtained the motion matrix as

$$
T=\left[\begin{array}{cccc}
\cos \theta & -\sin \theta & 0 & L-L \cos \theta \\
\sin \theta & \cos \theta & 0 & L(\cos 2 \theta-\tan \theta) \\
0 & 0 & 1 & 0 \\
0 & 0 & 0 & 1
\end{array}\right]
$$




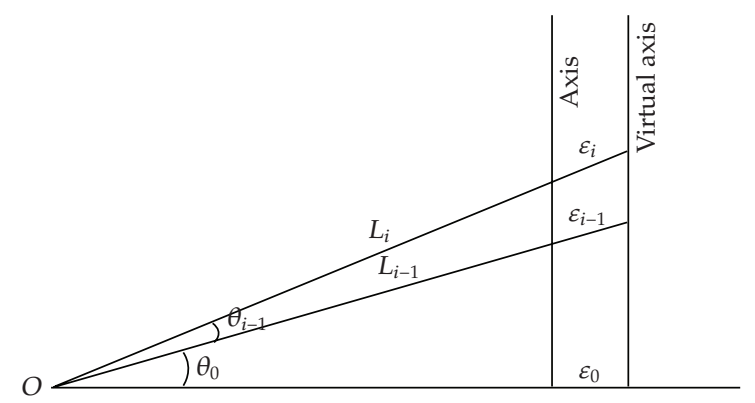

Figure 4: Relation between $L$ and $\varepsilon$ at any $t$ time.

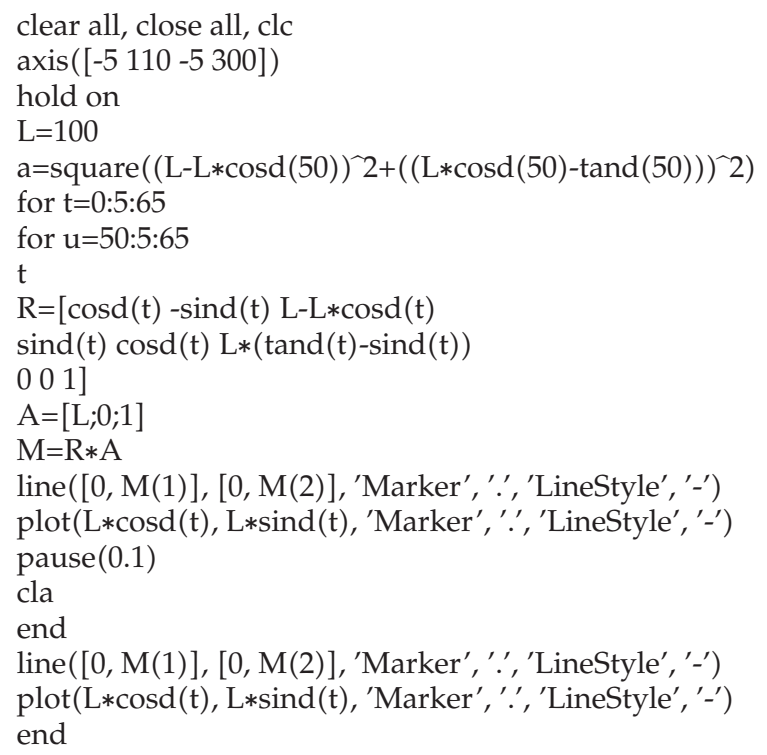

Algorithm 1

There is not any pressure to vertical axis in this machine as it is. If the machine is used with different purposes, such as painting, sandpaper, it must make a press to vertical axis.

While the output lengths of pistons are $L_{i}$, if the output of pistons are $L_{i}+\varepsilon_{i}$, the pistons make a pressure to a barrier. The fixed pressure belongs to the initial value which we determined. So

$$
\frac{L_{i-1}}{L_{i-1}+\varepsilon_{i-1}}=\frac{L_{i}}{L_{i}+\varepsilon_{i}}
$$

is obtained with Thales theorem and from Figure 4. 
Table 2: Correlation relation among $L_{i}, \omega_{i}$, and $\varepsilon_{i}$.

\begin{tabular}{|c|c|c|c|}
\hline & $L_{i}$ & $\omega_{i}$ & $\varepsilon_{i}$ \\
\hline & & & $\varepsilon_{0}=5$ \\
\hline 1 & 100 & 0,1 & 5,00076 \\
\hline 2 & 100,0152 & 0,1 & 5, 00305 \\
\hline 3 & 100,061 & 0,0999 & 5,00686 \\
\hline 4 & 100,1372 & 0,0999 & 5,01221 \\
\hline 5 & 100,2442 & 0,0998 & 5,0191 \\
\hline 6 & 100,382 & 0,0996 & 5,02754 \\
\hline 7 & 100,5508 & 0,0995 & 5,03755 \\
\hline 8 & 100,751 & 0,0993 & 5,04914 \\
\hline 9 & 100,9828 & 0,099 & 5,062325 \\
\hline 10 & 101,2465 & 0,0988 & 5,077135 \\
\hline 11 & 101,5427 & 0,0985 & 5,093585 \\
\hline 12 & 101,8717 & 0,0982 & 5, 111705 \\
\hline 13 & 102,2341 & 0,0978 & 5,13152 \\
\hline 14 & 102,6304 & 0,0974 & 5,15307 \\
\hline 15 & 103,0614 & 0,097 & 5,17638 \\
\hline 16 & 103,5276 & 0,0966 & 5, 201495 \\
\hline 17 & 104,0299 & 0,0961 & 5,22846 \\
\hline 18 & 104,5692 & 0,0956 & 5,25731 \\
\hline 19 & 105,1462 & 0,0951 & 5,288105 \\
\hline 20 & 105,7621 & 0,0946 & 5,32089 \\
\hline 21 & 106,4178 & 0,094 & 5,355725 \\
\hline 22 & 107,1145 & 0,0934 & 5,392675 \\
\hline 23 & 107,8535 & 0,0927 & 5,4318 \\
\hline 24 & 108,636 & 0,0921 & 5,47318 \\
\hline 25 & 109,4636 & 0,0914 & 5,51689 \\
\hline 26 & 110,3378 & 0,0906 & 5,56301 \\
\hline 27 & 111,2602 & 0,0899 & 5,61163 \\
\hline 28 & 112,2326 & 0,0891 & 5,66285 \\
\hline 29 & 113,257 & 0,0883 & 5,71677 \\
\hline 30 & 114,3354 & 0,0875 & 5,773505 \\
\hline 31 & 115,4701 & 0,0866 & 5,833165 \\
\hline 32 & 116,6633 & 0,0857 & 5,89589 \\
\hline 33 & 117,9178 & 0,0848 & 5,961815 \\
\hline 34 & 119,2363 & 0,0839 & 6,03109 \\
\hline 35 & 120,6218 & 0,0829 & 6,103875 \\
\hline 36 & 122,0775 & 0,0819 & 6,18034 \\
\hline 37 & 123,6068 & 0,0809 & 6,26068 \\
\hline 38 & 125,2136 & 0,0799 & 6,34509 \\
\hline 39 & 126,9018 & 0,0788 & 6,4338 \\
\hline 40 & 128,676 & 0,0777 & 6,527035 \\
\hline 41 & 130,5407 & 0,0766 & 6,625065 \\
\hline 42 & 132,5013 & 0,0755 & 6,728165 \\
\hline
\end{tabular}


Table 2: Continued.

\begin{tabular}{lccc}
\hline & $L_{i}$ & $\omega_{i}$ & $\varepsilon_{i}$ \\
\hline 43 & 134,5633 & 0,0743 & 6,836635 \\
44 & 136,7327 & 0,0731 & 6,95082 \\
45 & 139,0164 & 0,0719 & 7,07107 \\
46 & 141,4214 & 0,0707 & 7,197785 \\
47 & 143,9557 & 0,0695 & 7,331395 \\
48 & 146,6279 & 0,0682 & 7,472385 \\
49 & 149,4477 & 0,0669 & 7,621265 \\
50 & 152,4253 & 0,0656 & 7,77862 \\
51 & 155,5724 & 0,0643 & 7,94508 \\
52 & 158,9016 & 0,0629 & 8,121345 \\
53 & 162,4269 & 0,0616 & 8,3082 \\
54 & 166,164 & 0,0602 & 8,50651 \\
55 & 170,1302 & 0,0588 & 8,717235 \\
56 & 174,3447 & 0,0574 & 8,94146 \\
57 & 178,8292 & 0,0559 & 9,18039 \\
58 & 183,6078 & 0,0545 & 9,4354 \\
59 & 188,708 & 0,053 & 9,70802 \\
60 & 194,1604 & 0,0515 & 10 \\
61 & 200 & 0,05 & 10,313325 \\
62 & 206,2665 & 0,0485 & 10,65027 \\
63 & 213,0054 & 0,0469 & 11,013445 \\
64 & 220,2689 & 0,0454 & 11,40586 \\
65 & 228,1172 & 11,83101 \\
66 & 236,6202 & 0,0438 & 11,83101 \\
\hline
\end{tabular}

At the same time, the following equalities are obtained with information from Figure 4.

$$
\begin{aligned}
& L_{i}=\frac{L_{0}}{\cos \theta_{i}}, \\
& \| \overrightarrow{O P_{y_{i}} \|}=L \tan \theta_{i}, \\
& w_{i}=\frac{v}{L_{i}}, \\
& \varepsilon_{i}=\frac{L_{i}}{L_{i-1}} \varepsilon_{i}-1, \\
& L_{i} w_{i}=v: \text { constant } \\
& \varepsilon_{i}=\frac{w_{i}-1}{w_{i}} \varepsilon_{i-1} .
\end{aligned}
$$

These equalities are used at correlation between $L, w$ and $\varepsilon$. 


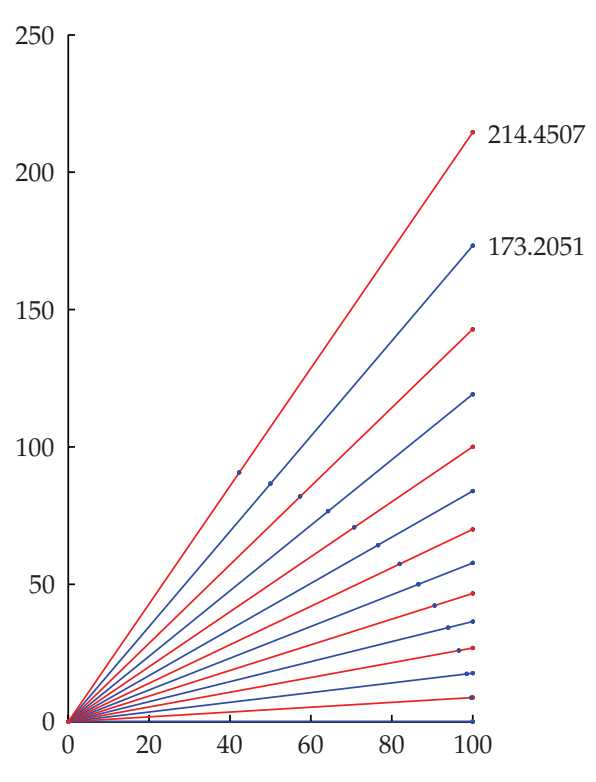

Figure 5

$\operatorname{cor}(\varepsilon, w)$ approaches to -1 from

$$
\varepsilon_{i} w_{i}=w_{i-1} \varepsilon_{i-1} .
$$

$\operatorname{cor}(\varepsilon, L)$ approaches to 1 because

$$
\frac{\varepsilon_{i}}{\varepsilon_{i-1}}=\frac{L_{i}}{L_{i-1}}
$$

$\operatorname{cor}(L, w)$ approaches to -1 because

$$
L_{i} w_{i}: \text { constant. }
$$

\section{Experimental Result and Discussions}

For $L=100, \varepsilon_{0}=5,0 \leq \theta \leq 65^{\circ}$, we calculate $L, \varepsilon_{i}$, and $w_{i}$ at Matlab. The programme at Matlab and its figure (Figure 5) are as Algorithm 1.

If correlation is calculated between $\varepsilon, w$, and $L$ values,

$$
\begin{gathered}
\operatorname{cor}(\varepsilon, \omega)=-0,976350874 \\
\operatorname{cor}(\varepsilon, L)=0,999677409 \\
\operatorname{cor}(L, \omega)=-0,975049457
\end{gathered}
$$

are obtained. This is a desired result. 


\section{Conclusions}

In this paper, RPPP mechanism, in which we used kinematical equations and we gave a design and a numeric sampling, is a prototype for a pressing. The machine is apt for being used especially in the specialized aims.

The pistons conducting translation are of hydraulic structure and should be considered to be controlled with double sensors. And finally, if we add $\varepsilon$ to $L$, then we can make a little press to fixed axis. Results of significance tests between $L, \varepsilon$ and $w$ are appropriate for the expectations. While $\varepsilon$ and $w$ have positive correlation, both $(\varepsilon, L)$ and $(L, w)$ have negative correlation but significant.

\section{References}

[1] S. B. Niku, Introduction to Robotics, Prentice Hall, Upper Saddle River, NJ, USA, 2001.

[2] K. S. Fu, R. C. Gonzalez, and C. S. G. Lee, Robotics, Mc Graw-Hill, Singapore, 1987.

[3] J. M. McCarthy, Geometric Design of Linkages, vol. 11 of Interdisciplinary Applied Mathematics, Springer, New York, NY, USA, 2000.

[4] R. L. Williams and B. H. Shelley, "Inverse kinematics for planar parallel manipulators," in Proceedings of the ASME Design Technical Conferences, Sacramento, Calif, USA, September 1997.

[5] J. Gadek, "3-parametric robot manipulator with intersecting axes," Applications of Mathematics, vol. 40, no. 2, pp. 131-145, 1995. 


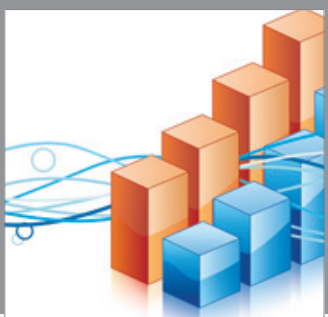

Advances in

Operations Research

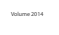

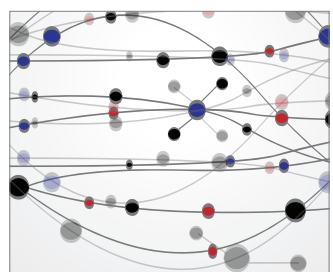

\section{The Scientific} World Journal
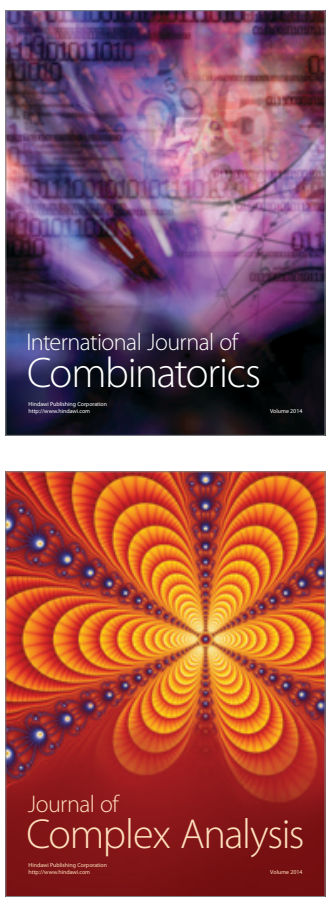

International Journal of

Mathematics and

Mathematical

Sciences
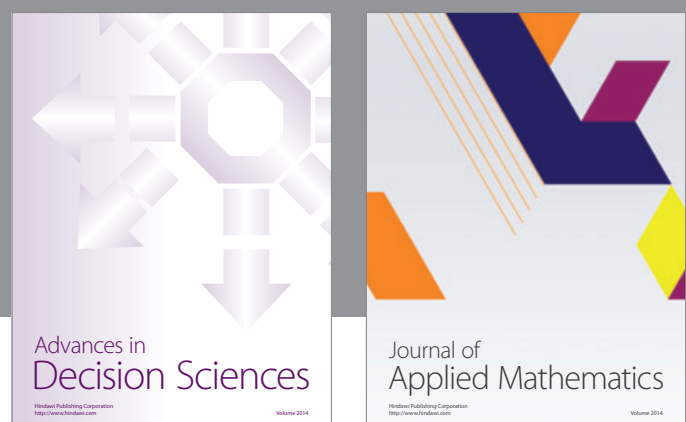

Journal of

Applied Mathematics
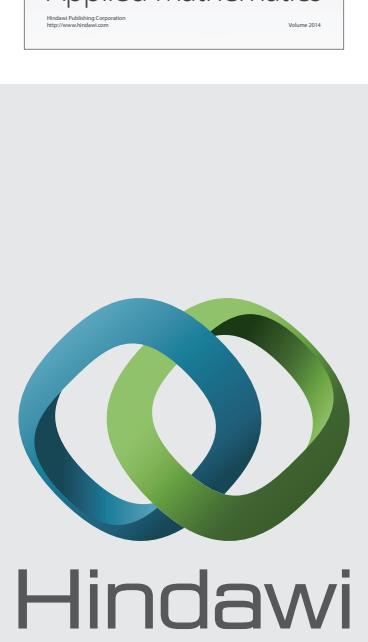

Submit your manuscripts at http://www.hindawi.com
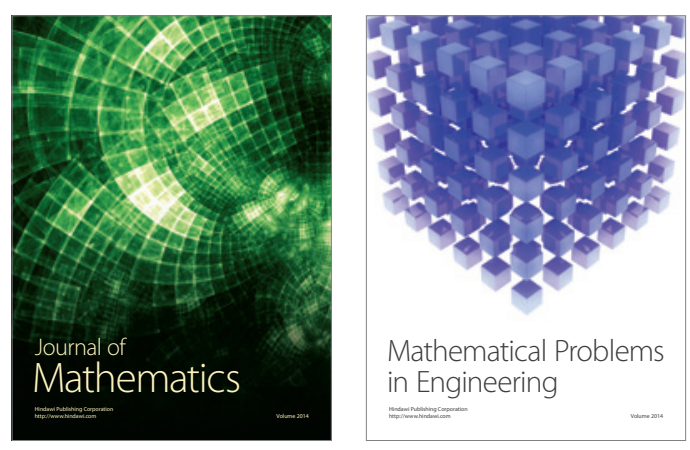

Mathematical Problems in Engineering
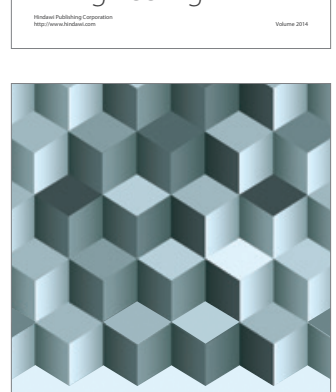

Journal of

Function Spaces
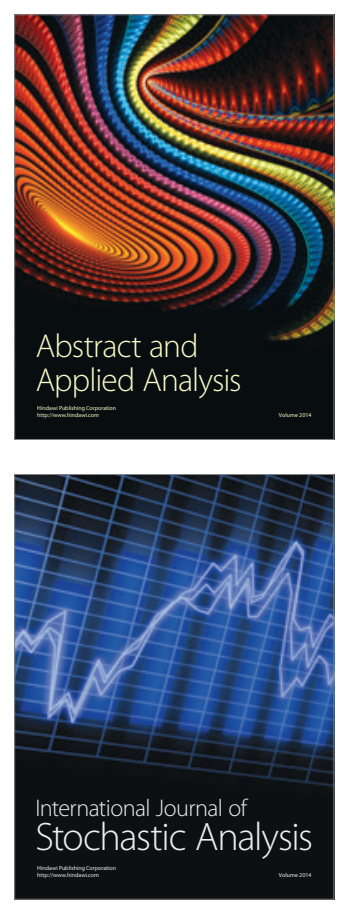

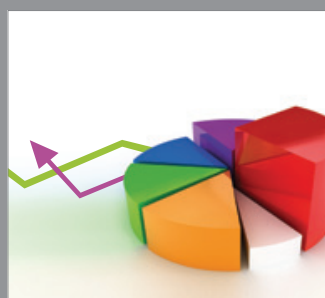

ournal of

Probability and Statistics

Promensencen
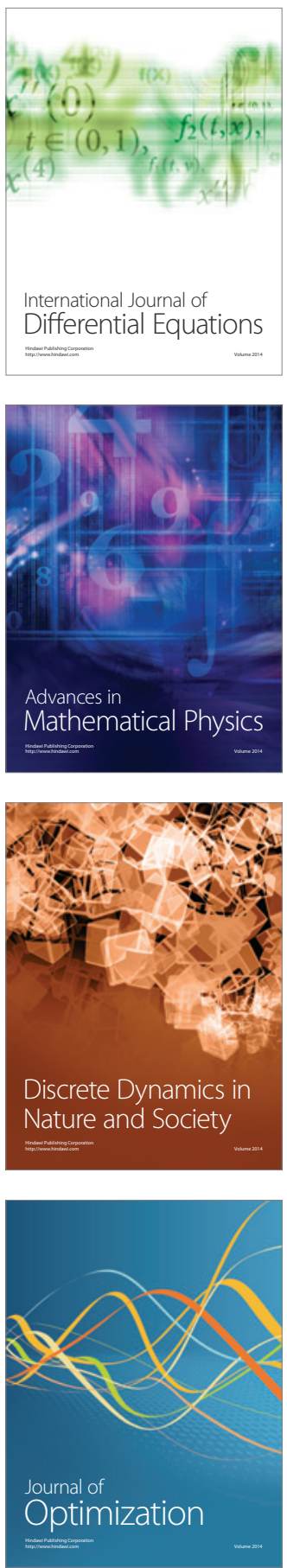